\title{
DEVELOPING A CO-DESIGN PROCESS MODEL FOR THE DIGITAL PRESENTATION OF INTANGIBLE CULTURAL HERITAGE: A CASE STUDY OF "WARM INHERITORS DIGITAL DIABOLO"
}

\author{
Cuiting Kong, Lie Zhang * \\ Academy of Arts \& Design, Tsinghua University, Beijing, China \\ kct18@mails.tsinghua.edu.cn, zhlie@tsinghua.edu.cn
}

KEY WORDS: Intangible Cultural Heritage, Co-design, Design Process, Virtual Reality, Interactive Digital Storytelling, Diabolo

\begin{abstract}
:
As digital technologies are becoming gradually integrated into museums and the preservation of intangible cultural heritage (ICH), museums and the exhibitions of ICH are becoming more attractive and flexible. However, digital technologies may also bring some problems, such as cultural alienation. The participation of the inheritors and communities of ICH in the design of digital exhibitions could reduce such problems. The main contribution of this paper is a co-design process model for digital exhibitions of ICH. The study was conducted by the project, "Warm Inheritors Digital Diabolo", which aimed to enhance the digital experience of diabolo by using virtual reality technology to implement interactive digital storytelling techniques. This project involved both designers and inheritors to realize the principle of respectful design. The results demonstrated the crucial role of inheritors and communities in the design process. This paper also offers some design recommendations.
\end{abstract}

\section{INTRODUCTION}

Intangible cultural heritage (ICH) refers to "the practices, representations, expressions, knowledge, skills-as well as the instruments, objects, artifacts, and cultural spaces associated therewith - that communities, groups, and, in some cases, individuals, recognize as part of their cultural heritage" (UNESCO, 2003). As a mainspring of cultural diversity and human creativity, ICH needs to be effectively safeguarded and passed on to future generations. The digitization of ICH is one of the most significant approaches to such protection.

Digital research on ICH includes the collection, storage, processing, presentation, and dissemination of knowledge in digital formats used to transform, reproduce, and restore ICH (Ma et al., 2019). As an essential part of the protection of $\mathrm{ICH}$, its presentation aims to show its value to the public and increase awareness for the need to protect and pass on ICH. Emerging digital media technologies, such as virtual reality (VR), augmented reality (AR), holographic projection, and embodied interaction, bring new possibilities to the presentation and dissemination of $\mathrm{ICH}$.

Digital media technology enables an audience anywhere in the world to experience $\mathrm{ICH}$ at any time, thus helping to attract more younger generations to traditional culture. However, the "living" and "intangible" characteristics of ICH leave it more vulnerable than tangible cultural heritage to disappearing. Inappropriate usage of digital media or improper interventions by external researchers and designers may affect the viability of $\mathrm{ICH}$. UNESCO emphasizes the core position of communities in the protection of ICH: "Communities, groups, and, where applicable, individuals should have the primary role in safeguarding their own intangible cultural heritage" (UNESCO, 2015). Community participation in the design process can reduce ethical problems. Therefore, inheritors and communities should participate in the design of digital exhibitions of ICH while considering the experience of the audience. Interactive digital storytelling (IDS) helps designers to transmit information about $\mathrm{ICH}$.
Recent years have seen an upsurge in ICH digital research but still not much discussion community participation in the design of digital exhibits. The main contribution of this paper is a new co-design process model for presenting ICH through VR and IDS, as seen in the case study of "Warm Inheritors Digital Diabolo".

\section{RELATED WORKS}

\subsection{Digital presentation of ICH}

Since UNESCO formally proposed to safeguard ICH in 2003, it has gradually attracted the attention of researchers and has stimulated intense academic discussions. The i-Treasure project (Cozzani et al., 2017) aims to identify and apply new digital tools, as well as define proper approaches to safeguarding and transmitting ICH. This project concentrates on four cases: rare singing, rare dancing, craftsmanship, and contemporary music composition. The Mingei projects (Partarakis et al., 2021) explore the possibilities of the digital preservation and presentation of Heritage Crafts (HCs), which involve skills, artifacts, materials, and tools. The MUSE research project (Khan, 2014) uses a VR simulation to teach audiences about the Al Ardha dance from the Middle East.

Research on the presentation of ICH in China has also made substantial progress. For example, Lu et al. ( 2011) introduced an interactive system to restore traditional Chinese shadow puppet theatre known as Piying. Deng et al. (2021) described a VR platform for conservating the ICH of the $\mathrm{Li}$ ethnic group in Hainan. Zhang et al. (2018) transferred the tacit knowledge of Huayao cross-stitching, an ethnic ICH in Hunan, into a digital learning format for rural children.

The above studies have focused on the digital collection, storage, and display of specific types of ICH. They have mainly discussed the use of digital technology (such as motion capture technology and databases) and digital presentation methods (such as serious game design and interactive narratives).

\footnotetext{
* Corresponding author
} 


\subsection{Community-based designs}

Tangible cultural heritage exhibitions usually focus on cultural relics and sites. Designers rely mainly on reading archaeological documents, consulting experts on cultural relics, visiting museums, and collecting network information for cultural research, as well as for inspiration. However, ICH exhibitions focus on people and activities. Designers need to enter the lifeworlds of $\mathrm{ICH}$, communicate with the inheritors and communities, and collect fresh stories about ICH. They also need to consider ethical problems and issues regarding value orientations.

Ethical frameworks for research in indigenous contexts require the participation of indigenous communities because researchers and designers should try to avoid the colonization of other cultures. Respect for Indigenous Knowledge (IK) regards the concern shown by researchers when they identify, explore, and assess the meaning of IK. "Respectful Design" (Sheehan, 2011) requires designers to treat any colonization crisis with broader, deeper, more comprehensive, and more cautious approaches to design. Cultural inheritors play a vital role in ICH. They have the right to join the digital storytelling process and express their ideas The participation of inheritors and communities in the design process helps increase cultural awareness and enhance cultural confidence. The Namibia University of Science and Technology (NUST) has conducted several long-term community-based design projects (Winschiers-Theophilus et al., 2010) to collect, store, present, and disseminate ICH by digital technology in cooperation with indigenous communities in Namibia, Africa. The Institute of Social Informatics and Technological Innovation (ISITI), Universiti Malaysia Sarawak (UNIMAS), has also launched some co-design projects (Zaman et al., 2016) with indigenous communities in Long Lamai, Malaysia, to explore digital preservation methods for ICH. Kingston University London and the City for Scientific Research and Technological Applications (SRTA-City) in Alexandria, Egypt, jointly organized a summer-school project (Giglitto et al., 2019) and recruited community members from different tribes of Bedouins settled in Borg El-Arab, Alexandria. Those community members cooperated with eighteen engineering students to document their ICH with mobile technologies.

These studies have focused on digitizing IK in specific regions, emphasizing the adjustment and combination of traditional participatory designs (PDs) and user-centered designs (UCDs) to develop new design methods that meet the needs of different design contexts and cultural backgrounds according to regional socio-technical and local interaction protocols.

\section{PROJECT BACKGROUND}

Diabolo is a traditional folk sport in China. With a long history of at least 900 years of spontaneous retention (Zhang, 2011), it has many regional variants, known by different names, such as "Kong Zhu," "Kong Zhong," "Dou Weng," "Feng Hulu," and "Che Ling". Included in the first list of state-level ICH in 2006, the sport possesses significant historical, cultural, and esthetic values. Diabolo used to be very popular in many cities, including Beijing. The regional variants cover wide and unique ranges of skills. However, most people passionate about the sport are middle-aged and senior adults, so this form of ICH has a lack of young inheritors to continue it. To encourage more younger people to learn about and participate in traditional diabolo, "Warm Inheritors Digital Diabolo" was launched as a co-design project funded by the National Social Science Foundation of
China to explore digital solutions for presenting and disseminating information about the sport.

\section{CO-DESIGN PROCESS}

As shown in Figure 1, the whole participatory design process was divided into three stages: (A) initial contact and embodied research, (B) formal cooperation and interactive narratives, and (C) common reflection and test iterations. The entire team was constituted as a bottom-up organization, this kind of organizational model is beneficial to balancing power among stakeholders while encouraging the designers and inheritors to make spontaneous contributions. The authors first established contact with the inheritors, then obtained support from the Beijing Diabolo Museum authorities and held a design workshop at the museum with more inheritors and designers participating in the design process.

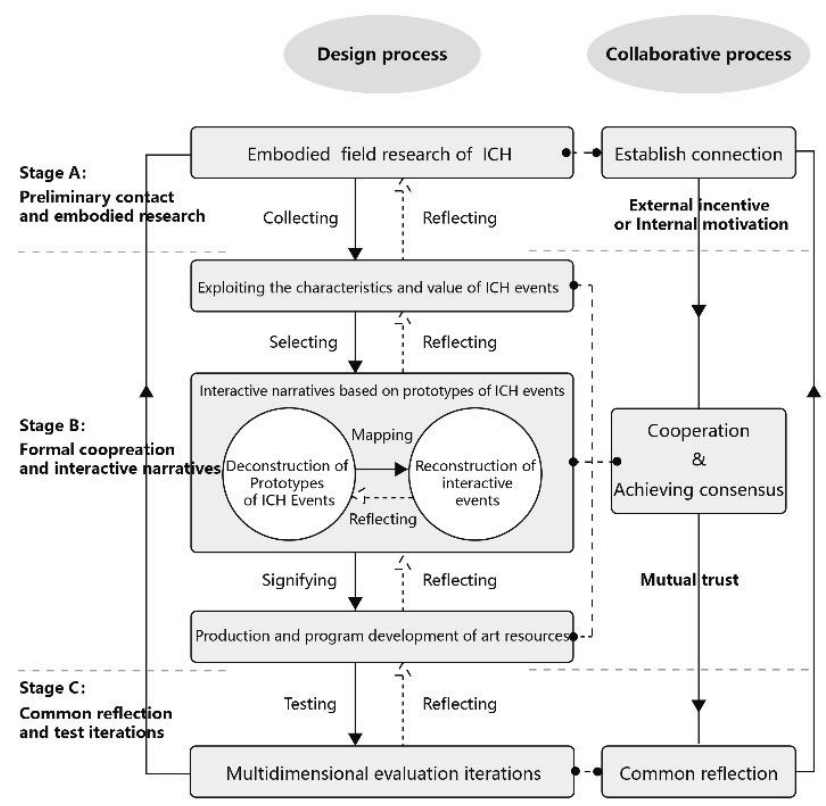

Figure 1. Co-design Process Model of Digital Presentation of ICH. Source: Authors

\subsection{Stage A: Preliminary contact and embodied research}

During cultural investigation, designers should not be satisfied with merely collecting information from the internet and literature but should also try to contact the inheritors and communities for instruction in diabolo, understand the related historical and cultural backgrounds, and collect fresh stories of recent and current diabolo events. The embodied experiences of the researchers are crucial to ethnographic fieldwork and consistent with the view of embodied ethnography (Turner, 2010).

The authors first established a partnership with an inheritor, Zhang, who is a volunteer guide of the Beijing Diabolo Museum, had inherited his expertise from his teacher and had been officially designated the ICH Inheritor of diabolo. He was very willing to participate in the project.

Many diabolo cultural relics are on display in the Beijing Diabolo Museum, which is a community museum managed by the authorities of Guangnei Street in Xicheng District and was specially designed to explore, display, disseminate, and protect diabolo culture. Several diabolo inheritors and enthusiasts have 
formed a team of volunteer guides in the museum to explain the history of diabolo to visitors and teach them how to play it.

To collect data on cultural relics and organize a design workshop at the museum, The authors submitted a project cooperation plan and obtained the permission and support from the museum and Guangnei Street authorities. Further inheritors and designers have joined the design process. Thus, a formal cooperative relationship was established with the external designer, the inheritors, and the museum.

\subsection{Stage B: Formal cooperation and interactive narratives}

To strengthen the sense of participation, The authors organized and established a "Warm Inheritors" design team and designed the visual system of the organization (Figure 2). The design team was composed of six inheritors and five designers (i.e., two graduate students and three Ph.D. students majoring in design). Collaborative design requires a common short-term goal, so the team set a phased goal to display the design results to the public at the museum during the Diabolo Cultural Festival.
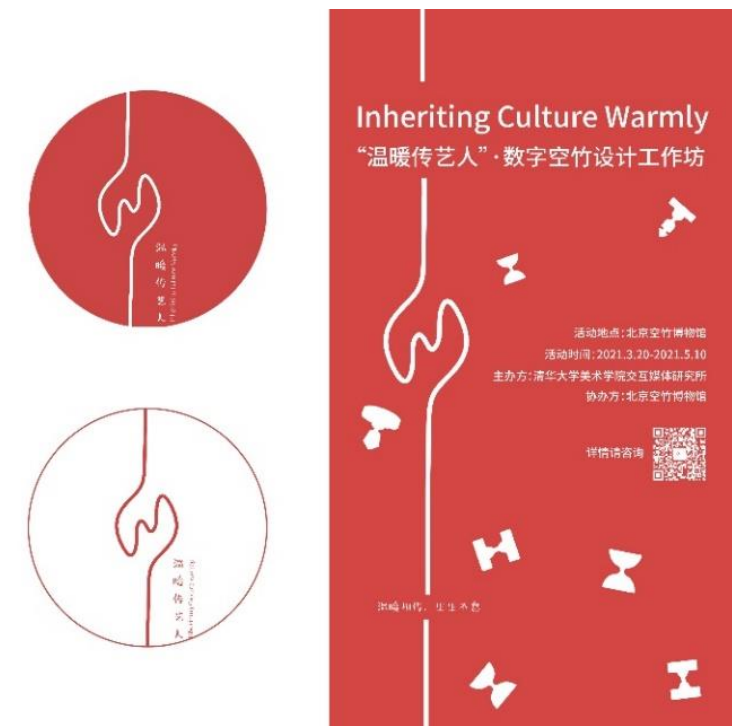

Figure 2. Visual System of "Warm Inheritors". Source: Authors

\subsubsection{Organization of design workshop}

Each team member received a toolkit, which included a work card, a blank Post-it Note for diabolo events, and another blank note for exhibition ideas. The first note was used to record the events or knowledge points that interested the member and would be introduced to the audience. The second note was used to record their ideas on presenting the events. Their ideas were then shared with the other members. The workshop started with mutual introductions of the participants and a brief introduction to the digital exhibition design cases. Then, the inheritors introduced the history of diabolo to the designers and taught them how to play. Finally, all the members discussed their ideas (figure $3)$.

The authors found that the designers were at ease with recording their ideas by writing or drawing on the notes, whereas the inheritors had difficulty doing the same but were better able to present their ideas through verbal instruction and physical movements. However, the sketches presented by the designers helped the inheritors to formulate their ideas and suggestions. The authors used photographs, video and audio recordings, and notes to record the entire process, then summarized the regional characteristics and value of diabolo through content analysis.

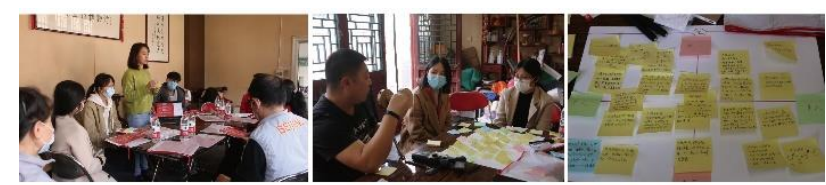

Figure 3. Design Workshop. Source: "Warm Inheritors" Team

\subsubsection{Exploiting the characteristics and value of ICH events}

The essential purpose of a physical or virtual exhibition is to explain an ICH's value and regional characteristics to the public. Therefore, a crucial step in the design process is to explore the characteristics and value of diabolo with the inheritors. After the content analysis, the team extracted five main characteristics of diabolo.

Artistic appreciation: reflected in the creativity and narrative of its performance. Diabolo players display many patterns of movement, which have folk names with meanings such as "immortal crossing bridge", "high mountains and flowing water", "watching the moon from left to right", and "two dragons playing with pearls". In addition, the decorations of diabolos are vibrant and reflect their creators' inner yearnings and wisdom. The patterns on the diabolos produce rich visual effects when rotated.

Competitiveness: competitive variants of diabolo, such as diabolo balls and diabolo competitions (e.g., how many "leg strings" can be completed in a minute), have emerged.

Suspense: diabolo is often seen as an acrobatic activity. The audience is often left in suspense to see if a player can accomplish a difficult maneuver.

Surprising diversity: a wide range of diverse skills, including single or double round, special-shaped diabolos, and single or multiple diabolos, can be exhibited. Skilled players can play with various types and sizes of diabolos. Various props, such as bottles, stools, handkerchiefs, and other everyday items, can be incorporated.

Sociality: some skills require two or more players. Such performances are challenging to perform and enjoyable to watch. Multiple players can cooperate to complete a task, which could increase the interactions between players.

\subsubsection{Interactive narratives based on prototypes of ICH events}

After analyzing the characteristics of $\mathrm{ICH}$, the team clarified the aim of this project to be the reflection of the rich imagination of diabolo practitioners and the knowledge of the sport passed from generation to generation. The project was to encourage more people to learn about diabolo, as well as to participate in the protection and transmission of diabolo culture. The design process required the selection and organization of materials based on the purposes and directions of the exhibitions to formulate prototypes of ICH events, which would provide the original forms of the events to be represented by digital media. The selected prototypes included the teaching and learning of the skills for playing diabolo, the production of diabolos, the collection of various types of diabolos, and the performance of diabolo demonstrations (Figure 4). 


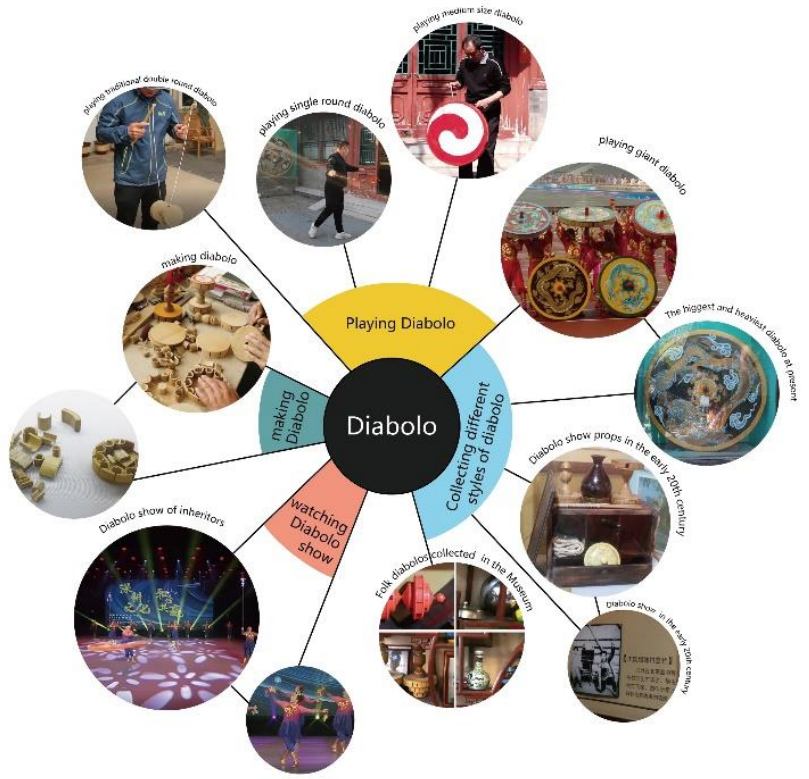

Figure 4. Prototypes of Diabolo Events. Source: Authors

The original ICH events occur at different times and spaces with non-structural relationships between the events. Through the narrative of digital media, the original events can be integrated into virtual spaces and revealed to the audience with specific temporal and logical semantic relationships. The audience understands the meaning by the interaction.

In the "Warm Inheritors Digital Diabolo" project, the authors initially conceived of a modern stage scene. However, later during cooperation with the inheritors, they proposed to take a quadrangle of Beijing as an interactive scene to better convey the regional characteristics of diabolo. The Beijing Diabolo Museum itself is located in a quadrangle. Virtual and physical experiences can form a mapping relationship. Therefore, the team finally used the quadrangle of the Beijing Diabolo Museum as the prototype of the virtual scenes and determined the following five scenes that were incorporated into the entire VR digital display (Figure $5-7)$.

Scene 1: an animated character plays diabolo in a virtual courtyard, which can be viewed from 360 degrees. The audience can also use a VR controller stick to play virtual diabolo, of which the various forms available are: traditional two-wheeled diabolo, a medium-sized diabolo with a spiral pattern, and a giant diabolo with a diameter of $1.6 \mathrm{~m}$.

Scene 2: teaches how diabolos are made by a diagram detailing the production process. All component structures are presented in front of the audience. The audience is instructed to use a VR controller stick to simulate the diabolo assembly process.

Scene 3: a game in which the audience collects diabolo relics. This game is based on the prototype of actual cultural relics, such as the Wang Sisters' diabolo props and box, in addition to different types of diabolos. The audience is instructed to search for and select the props of the Wang Sisters from many different diabolos. These props are then placed into the box to complete the task. Searching and comparing compel the audience to pay closer attention to the different diabolo styles and to understand the folk adage, "Everything can be played as a diabolo".
Scene 4: A display screen depicts a performance by the Guangnei Street Diabolo Art Troupe while two other screens play video clips collected from the audience, who have been encouraged to participate in the contribution of content about diabolo.

Scene 5: A message board in a room displays the whole process of designing the VR experience and instructs the audience to think of how they would have designed it.

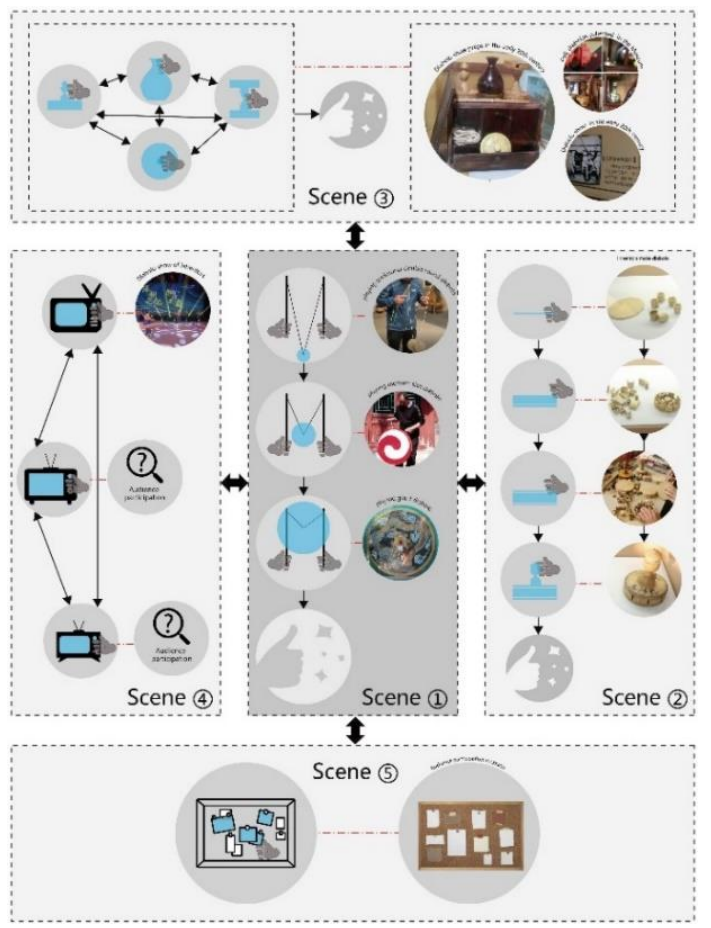

Figure 5. Reconstruction of Interactive Events. Source: Authors

The reconstructed diabolo interactive narrative experience integrates all the ICH event prototypes into a virtual quadrangle. Five spaces correspond to five storylines in which a audience can virtually walk and interact to promote the development and completion of the tasks in each storyline. This kind of plot structure is an anemone-type structure (Ryan, 2015), which is suitable for exhibitions at museums. The center of the courtyard is the core event space, which is connected to four rooms. The five storylines are independent in time sequence, so the audience can choose to enter any room. If the audience decides to stop the experience halfway, they would not affect the next audience's experience.

The team took full advantage of the capabilities of VR and formed a complementary relationship with a real visit to a diabolo museum. Instead of copying the exhibition mode and experience of a real museum, the team provided the audience with more exciting experiences, such as playing with different types of diabolos and the largest and heaviest diabolos, that would be impossible to offer in real museums due to its difficult skills and a certain degree of danger. The game mechanism adds more challenge and a sense of achievement to the experience. Moreover, virtually playing diabolo can be accompanied by the gorgeous visual effect of the movements, thus enhancing the enjoyment of the audience.

\subsubsection{Production and program development of art resources}

After the narrative framework and interactive events were defined, the logical architecture of the program was completed 
and the visualization work for defining the visual style of the whole scheme, including scenes, characters, and interactive interfaces, was started. The team adopted the exquisite Chinese meticulous hand-painted style to bring a unique experience to the audience (Figure 6).

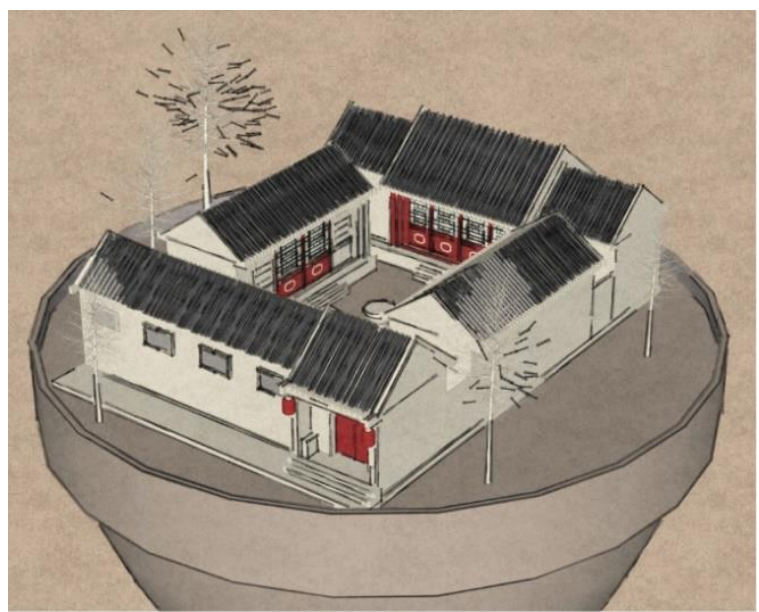

Figure 6. VR Scenes. Source: "Warm Inheritors" Team

After the program's visual designs of the art assets were completed, data, which included movements, cultural relics, and sounds, were collected. The authors invited the inheritors to capture the motion data and sounds in a motion capture laboratory. Models of the scenes and characters were low poly models used to reduce the computational burdens on the system and create a smooth experience for the audience. The character models were bound to skeletons, paired with the collected motion data, and adjusted for animation. The completed art assets were uploaded into the Unity3D platform (Figure 7).

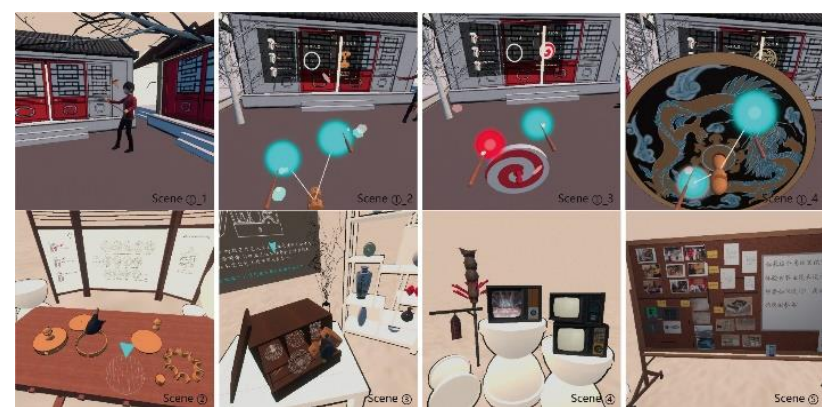

Figure 7. VR Interfaces. Source: "Warm Inheritors" Team

\subsection{Stage C: Common reflection and test iterations}

After the application's development is complete, inheritors will be invited to test and evaluate the design: (1) if the interactive experience is natural and smooth, and (2) if it accurately and effectively presents the characteristics and value of diabolo. After approval, the application will be presented to the public. This stage will be attempted in a future study.

\section{DISCUSSION AND FUTURE RESEARCH}

This study explored a new design process model of ICH digital exhibition design based on participatory design methods and interactive digital storytelling. The authors believe that depicting the "living" characteristics of ICH is essential to its digital exhibition. The co-design process model can guide inheritors and designers to join the design process and better realize respectful design and technological democracy.
The authors were fortunate to have met some inheritors of diabolo who were very interested in the project and very enthusiastic about transferring their knowledge. They actively joined the design process and provided many creative ideas. However, encouraging the participation of more inheritors was a problem. What is the root of the continuous dynamic mechanism for inheritors to participate in the design process? Which tools can be used to promote the long-term sustainable participation of inheritors?

Projects supported by national funds are considered as public welfare projects, so the intellectual property rights should belong to the state while all participating personnel have the right of signature and display. Project funds provide labor remuneration to every member who contributes to the project. However, once the project is completed, the continuation of participatory activities would be difficult without further financial support. Any economic benefits generated from the design would contribute to the project's sustainable development, but a marketoriented design would involve more complex issues regarding the design's intellectual property rights, which significantly affect the depth of the cooperation by the stakeholders. Solutions to problems involving intellectual property rights will be addressed in follow-up research.

\section{CONCLUSION}

For the digital presentation of ICH, the participation of inheritors and communities in the design process is quite necessary to ensure their discourse rights, enhance their cultural confidence, and promote the inheritance and sustainable development of ICH. The authors have formulated a co-design process model for the digital presentation of ICH based on the "Warm Inheritors Digital Diabolo" project. The authors divided the design process into three stages: (A) initial contact and embodied research, (B) formal cooperation and interactive narratives, and (C) common reflection and test iterations. In each stage, the role positioning of and organizational relationships among the participants were changing. Community-based participatory design required the organizers to fully consider the interaction protocols of the participants in order to apply participatory design methods appropriate to the local cultural backgrounds.

The bottom-up design organization presented in this paper is suitable for China's policy of ICH protection by government guidance, social participation, diversified investment, and cooperative development. Inheritors and designers lead the design process and produce the results together. The collaboration on the design and the display of the final results occur at community museums. Audiences are encouraged to participate as much as possible in contributing content to ICH digital exhibitions. The authors hope that this study will inspire more research and stimulate more discussion on the respectful design of ICH exhibitions.

\section{ACKNOWLEDGMENTS}

The authors thank all the participants for their contributions and the Beijing Diabolo Museum for its support.

Funded by China's National Social Science Foundation, this study was a collaborative project involving digital data collection, the creation of an intelligent platform, and the global communication of China's intangible cultural heritage. (19ZDA336) 


\section{REFERENCES}

Cozzani, G., Pozzi, F., Dagnino, F. M., Katos, A. V.,Katsouli, E. F., 2017. Innovative technologies for intangible cultural heritage education and preservation: the case of i-Treasures. Personal \& Ubiquitous Computing, 21(2), 1-13.

Deng, X., Kim, I. T.,Shen, C., 2021. Research on Convolutional Neural Network-Based Virtual Reality Platform Framework for the Intangible Cultural Heritage Conservation of China Hainan Li Nationality: Boat-Shaped House as an Example. Mathematical Problems in Engineering, 2021, 1-16.

Giglitto, D., Lazem, S.,Preston, A., 2019. A Participatory Approach for Digital Documentation of Egyptian Bedouins Intangible Cultural Heritage. Interaction Design and Architecture(s) Journal, 41, 31-49.

Khan, M., 2014. MUSE: Understanding traditional dances, Virtual Reality, IEEE, Minneapolis, MN, USA, 173-174. dio.org/ 10.1109/VR.2014.6802107

Lu, F., Tian, F., Jiang, Y., Cao, X., Luo, W., Li, G., Zhang, X., Dai, G.,Wang, H., 2011. ShadowStory: creative and collaborative digital storytelling inspired by cultural heritage, CHI 2011, ACM, Vancouver, BC, Canada, 1919-1928. dio.org/10.1145/1978942.1979221

Ma, X. N., Tu, L.,Xu, Y. Q., 2019. Development status of the digitization of intangible cultural heritages. Scientia Sinica(Informationis), 49(2), 121-142.

Partarakis, N., Kaplanidi, D., Doulgeraki, P., Karuzaki, E., Petraki, A., Metilli, D., Bartalesi, V., Adami, I., Meghini, C.,Zabulis, X., 2021. Representation and Presentation of Culinary Tradition as Cultural Heritage. Heritage, 4(2), 612 640 .

Ryan, M., 2015: Narrative as Virtual Reality 2: Revisiting Immersion and Interactivity in Literature and Electronic Media. Johns Hopkins University Press, Baltimore.

Sheehan, N. W., 2011. Indigenous Knowledge and Respectful Design: An Evidence-Based Approach. Design Issues, 27(4), 68-80.

Turner, A., 2010. Embodied ethnography. Doing culture. Social Anthropology, 8(01), 51-60.

UNESCO, 2003: Convention for the safeguarding of the Intangible Cultural Heritage. https://ich.unesco.org/en/convention(2 July 2021).

UNESCO, 2015: Ethics and Intangible Cultural Heritage.http://ich.unesco.org/en/ethics-and-ich-00866(2 June 2021).

Winschiers-Theophilus, H., Chivuno-Kuria, S., Kapuire, G. K., Bidwell, N. J.,Blake, E., 2010. Being participated: a community approach, PDC '10: The 11th Biennial Participatory Design Conference, Association for Computing Machinery, Sydney, Australia, 1-10. dio.org/10.1145/1900441.1900443

Zaman, T., Yeo, A. W.,Jengan, G., 2016. Designing Digital
Solutions for Preserving Penan Sign Language: A Reflective Study. Advances in Human-Computer Interaction, 2016, 1-9.

Zhang, D. F., 2011. Diabolo and its path of development from a culture perspective. Journal of Shandong Institute of Physical Education and Sports, 10(27), 48-52.

Zhang, D., Yang, Y., Ji, T., Xie, H.,He, Y., 2018. Designing Craft Learning Experience for Rural Children: A Case Study on Huayao Cross-Stitch in Southwest China, 10th International Conference, CCD 2018, Held as Part of HCI International 2018, Springer International Publishing, Las Vegas, NV, USA, 117132. dio.org/10.1007/978-3-319-92252-2_9 